\title{
La pedagogía de la Compañía de Jesús ante los retos del mundo actual
}
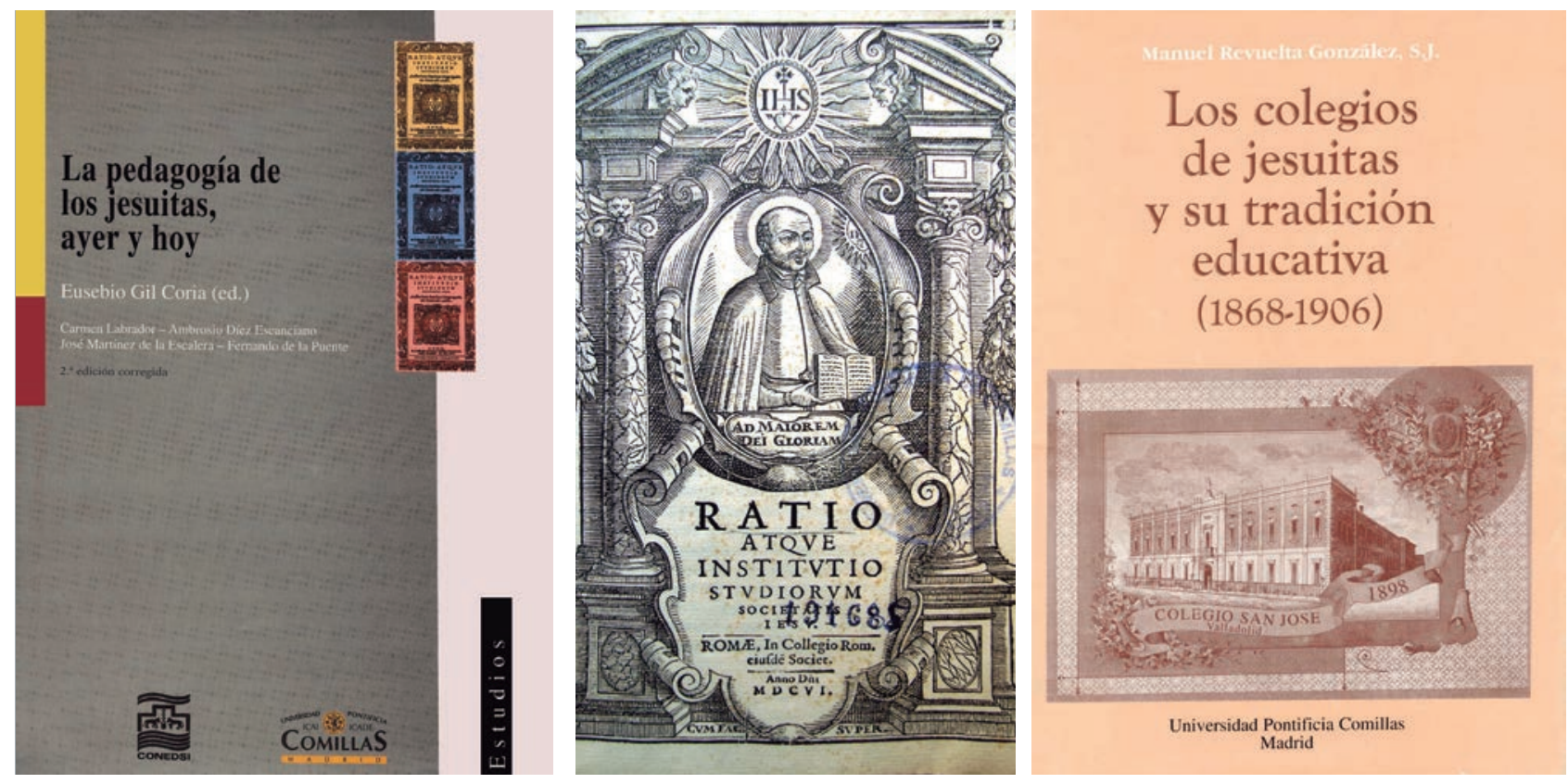

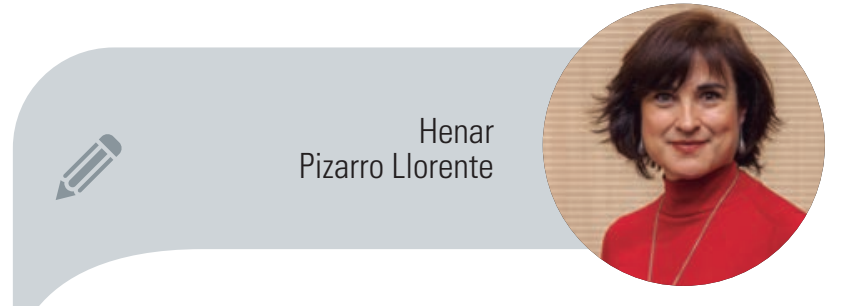

Universidad Pontificia Comillas

hpizarro@comillas.edu

\section{La vigencia de una tradición}

La Compañía de Jesús atesora una tradición centenaria en el ámbito de la educación. Desde su conformación en 1540, los jesuitas encontraron en la fundación y gestión de centros educativos una labor que, si bien no estaba contemplada entre sus ministerios iniciales, se convirtió en una de las señas de identidad del naciente instituto. Crearon un modelo educativo que implantaron en todos los colegios, basado en el método del "modus parisienses" y en el estudio de las humanidades, teniendo en cuenta que las mismas englobaban los estudios científicos. La gratuidad y la adaptación a las características y circunstancias de "lugares y personas" formaron parte del proyecto inicial.

El sistema pedagógico quedó fijado en la Ratio Studiorum, cuyo texto definitivo se publicó en 1599.

Su contenido no era doctrinal, sino que reunía las reglas prácticas que sintetizaban la experiencia docente acumulada en medio siglo. Sin duda, el proyecto respondió a las necesidades educativas de un mundo que se enfrentaba a la llamada "primera globalización", puesto que se ofrecía un plan de estudios unitario y universal que entretejía conexiones culturales entre los centros existentes en todo el mundo. EI P. Manuel Revuelta González nos ofreció su magisterio sobre estas cuestiones en esta misma sección ( $n$. $^{\circ}$ 341, octubre 2011), a cuyas páginas nos remitimos, dado que explican el plan de estudios, los métodos didácticos y los grandes principios pedagógicos.

Esta innovadora actividad tuvo un éxito considerable y alcanzó un indudable prestigio por la calidad de la enseñanza impartida, pero la expulsión y posterior disolución de la Compañía de Jesús quebraron el proyecto. La importancia adquirida por la dimensión educativa quedó reflejada en la bula de restablecimiento de la Compañía de Jesús otorgada por el papa Pío VII en 1814. Mientras que no se hizo referencia en el documento fundacional de la orden a esta cuestión, la dedicación de los jesuitas a la formación de la juventud aparecía significado como un ministerio prioritario en los albores del siglo XIX. No obstante, a pesar del entusiasmo con el que los jesuitas retomaron esta labor, las dificultades fueron muchas y de diversa consideración. La convulsión política de 


\section{José Alberto Mesa, SJ (ed.)}

\section{LA PEDAGOGÍA IGNACIANA}

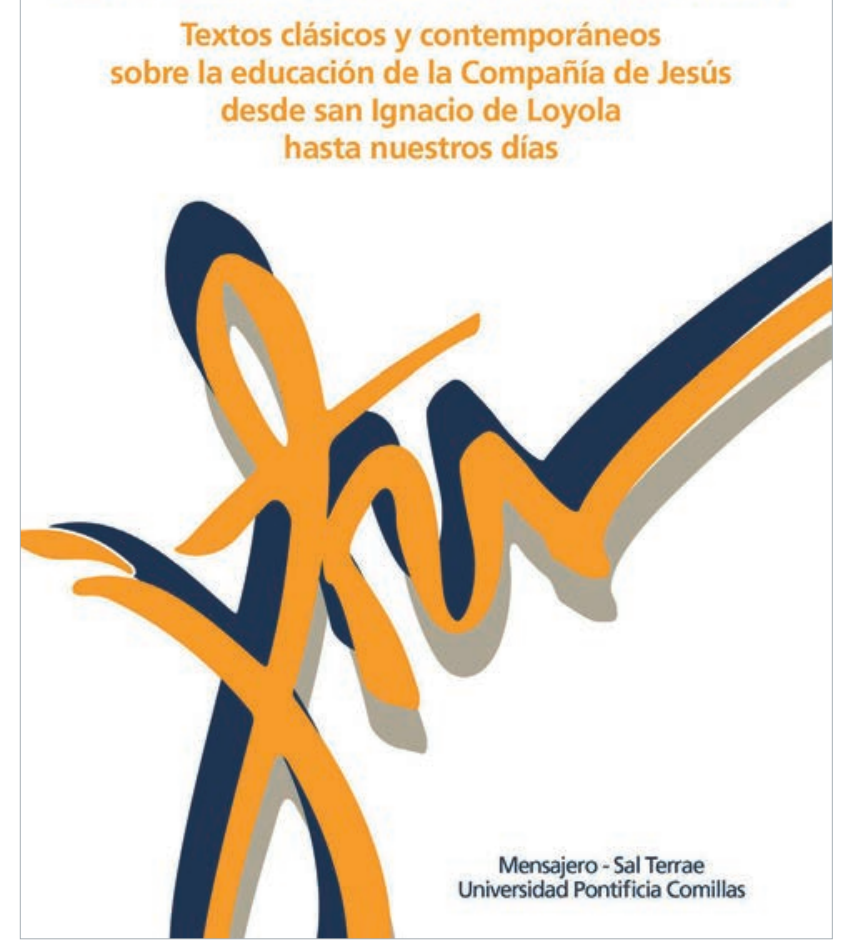

dicha centuria y de la siguiente, así como los avatares académicos que acompañaron este devenir en la mayoría de los países, supusieron un escenario marcado por la inestabilidad. Además, la conservación de la Ratio Studiorum se hizo inviable tanto para la validez de los grados como por los avances de los conocimientos, que exigía nuevas asignaturas y nuevos métodos pedagógicos que se fueron aplicando de manera discontinua y desigual. Nuevamente, el P. Manuel Revuelta González analizó estas vicisitudes en una magistral síntesis ( . $^{\circ} 346$, agosto 2012). No obstante, los centros educativos de la Compañía de Jesús continuaron teniendo en común la espiritualidad ignaciana, una alta exigencia académica y la formación integral de la persona.

\section{La pedagogía ignaciana}

En consonancia con lo dispuesto por el Concilio Vaticano II en materia educativa, los Padres Generales y las Congregaciones Generales señalaron que la tradición educativa debía de acomodarse a las nuevas circunstancias sin perder la raíz que la sustenta. En este sentido, cabe hacer referencia a dos documentos: Características de la educación de la Compañía de Jesús (1986) y Pedagogía ignaciana. Un planteamiento práctico (1993). Conviene resaltar la inclusión de los términos "pedagogía ignaciana", que comenzó a utilizarse en preferencia a "pedagogía jesuítica" en algunos ámbitos. Si hemos mantenido la fórmula en el título de estas páginas se debe a que pretenden ser una continuidad de los artículos precedentes citados del P. Revuelta y un pequeño homenaje a su magisterio tras su reciente fallecimiento.

El P. Arrupe afirmó que la escuela jesuita debía de dar a sus estudiantes una cierta "ignacianidad" o un modo de proceder según la visión de los Ejercicios Espirituales. Fue el inicio de una "fidelidad creativa", invocando a esa tradición de flexibilidad y respeto por el contexto, que se sintetizó en dar nitidez a la misión de educar "hombres y mujeres para los demás". La Compañía de Jesús ha redoblado sus esfuerzos por dar a conocer los principios de identidad de la pedagogía ignaciana adaptada a la realidad del mundo actual. Ciertamente, los documentos citados en las líneas anteriores han venido a convertirse en los sucesores de la Ratio Studiorum en la medida en que otorgaron la uniformidad al lenguaje y a la finalidad de la educación. En consecuencia, durante estos años, la mayoría de los centros educativos incorporaron programas nuevos, sustentados en la colaboración entre jesuitas y laicos, lo que ya supuso una innovación en sí misma, con el objetivo de favorecer la justicia social, el pensamiento crítico y la opción preferencial por los pobres. El referido documento publicado en 1993 es nombrado en ocasiones como Paradigma Pedagógico Ignaciano (incluso por las siglas PPI). La pedagogía activa propuesta se centra en cinco dimensiones orientadas a una buena práctica pedagógica. No se trata de una fórmula que anule la búsqueda de opciones de mejoras o aportaciones externas, pero sí supone un criterio para hacer una selección y como marco para orientar las relaciones entre maestros y estudiantes. Así, se promueve el respeto por el contexto del aprendizaje, se integran - a través de actividades prácticas - el conocimiento y la emoción para que resulten una experiencia significativa, se estimula la reflexión que permita entender y profundizar en el aprendizaje, se retoma la práctica que se transforma en el proceso y favorece la toma de decisión y el compromiso para, finalmente, poder evaluar el crecimiento real realizado por los alumnos no solo académico, sino también en el ámbito personal y humano.

Una fuente esencial para acercarse a la pedagogía ignaciana es el corpus constituido por los discursos publicados por los distintos Generales (Kolvenbach, Nicolás y Sosa). El P. Kolvenbach enlazaba con la tradición humanista cuando significó que había que educar "hombres y mujeres conscientes, competentes y con compromiso compasivo" (1993). El P. Adolfo Nicolás hizo referencia reiterada en sus intervenciones 


\section{[Texto 1]}

De acuerdo con la gran tradición jesuita, llega ahora el tiempo de la repetición —un resumen- He intentado reflexionar con vosotros sobre los retos de la globalización a las universidades jesuitas como instituciones de enseñanza, servicio e investigación. En primer lugar, en respuesta a la globalización de la superficialidad, sugiero que necesitamos estudiar más profundamente el mundo cultural emergente de nuestros estudiantes y encontrar formas creativas de promover la profundidad del pensamiento e imaginación; una profundidad que sea transformadora de la persona. En segundo lugar, para aumentar el potencial de las nuevas posibilidades de comunicación y cooperación, urjo a las universidades jesuitas a que trabajen para formar una red internacional operativa que se ocupe de temas importantes que conciernen a la fe, la justicia y la ecología, que son retos que trascienden países y continentes. Finalmente, para luchar contra la desigualdad en la distribución del conocimiento, animo a una búsqueda de formas creativas para compartir los frutos de la investigación con los excluidos; y en respuesta a la difusión global del secularismo y el fundamentalismo, invito a las universidades jesuitas a renovar el compromiso con la tradición jesuita del ministerio intelectual, que media entre la fe y la cultura.

\section{Comentarios para Redes para la educación superior jesuita: configurar un futuro para un mundo humano, justo Nicolás sostenible \\ Ciudad de México, 23 de abril de 2010}

\section{[Texto 2]}

Estos cuatro calificativos expresan la "excelencia humana" que la Compañía de Jesús quiere para los jóvenes que nos confía la sociedad: competentes, profesionalmente hablando, porque tienen una formación académica que les permite conocer con rigor los avances de la ciencia y de la tecnología; conscientes, porque además de conocerse a sí mismos, gracias al desarrollo de su capacidad de interiorización y al cultivo de la vida espiritual, tienen un consistente conocimiento y experiencia de la sociedad y de sus desequilibrios; compasivos, porque son capaces de abrir su corazón para ser solidarios y asumir sobre sí el sufrimiento que otros viven; y comprometidos, porque, siendo compasivos, se empeñan honestamente y desde la fe, y con medios pacíficos, en la transformación social y política de sus países y de las estructuras sociales para alcanzar la justicia.

P. Adolfo Nicolás

VIII Congreso de la Unión Mundial de Antiguos Alumnos Jesuitas Medellín (Colombia), 15 de agosto de 2013

\section{[Texto 3]}

La razón de ser de nuestros colegios es el servicio a la misión. Hoy esta misión implica un servicio de fe, justicia y cuidado del medio ambiente. Debemos asegurarnos de que nuestros colegios continúen enfocados en nuestra misión y puedan educar a la actual y a las próximas generaciones en nuestra tradición holística. Reconocemos que nuestros colegios sienten una tensión o experimentan una brecha entre servir a la misión y seguir siendo relevantes para los estudiantes, los padres de familia y la sociedad en general. Esta tensión siempre ha estado presente en nuestra historia y ha desafiado nuestra creatividad. El contexto actual requiere un serio discernimiento para garantizar que nuestra misión de reconciliación y justicia se refleje en nuestros colegios. Dado el advenimiento de la inteligencia artificial, la cuarta revolución industrial y sus implicaciones para la experiencia humana y los cambios que conllevarán en las condiciones de trabajo, incumbe a nuestros colegios arraigarse en la educación humanista por la que los colegios jesuitas han sido siempre reconocidos.

Acuerdos finales del Congreso Internacional de los Delegados de Educación de la Compañía de Jesús

Río de Janeiro, 2017

\section{[Texto 4]}

La interculturalidad nos hace vivir más plenamente la universalidad, pues acoge las diferencias culturales como revelación del rostro de la humanidad creada a imagen y semejanza de Dios, y se enriquece del intercambio cada vez más profundo entre ellas. La interculturalidad no es un fin en sí misma, sino el medio a través del cual creamos las condiciones para vivir plenamente la humanidad, contribuyendo a la humanización de las personas, las culturas y los pueblos. Es algo más que el reconocimiento de la existencia de muchas culturas, en el presente y en el pasado (multiculturalidad). Surge de la construcción de puentes y de comunicación fluida entre ellas. Proceso complejo, no exento de conflictos, que no es apenas un "encuentro entre culturas" para crear un espacio supra-, meta- o transcultural. Es más bien un "intercambio recíproco entre culturas que puede conducir a la transformación y el enriquecimiento de todos los implicados", pero sin excluir o sustituir la inculturación, y más bien profundizándola, porque nadie puede ofrecer a otros lo que no tiene.

Acuerdos finales del Congreso Internacional de los Delegados de Educación de la Compañía de Jesús Río de Janeiro, 2017 

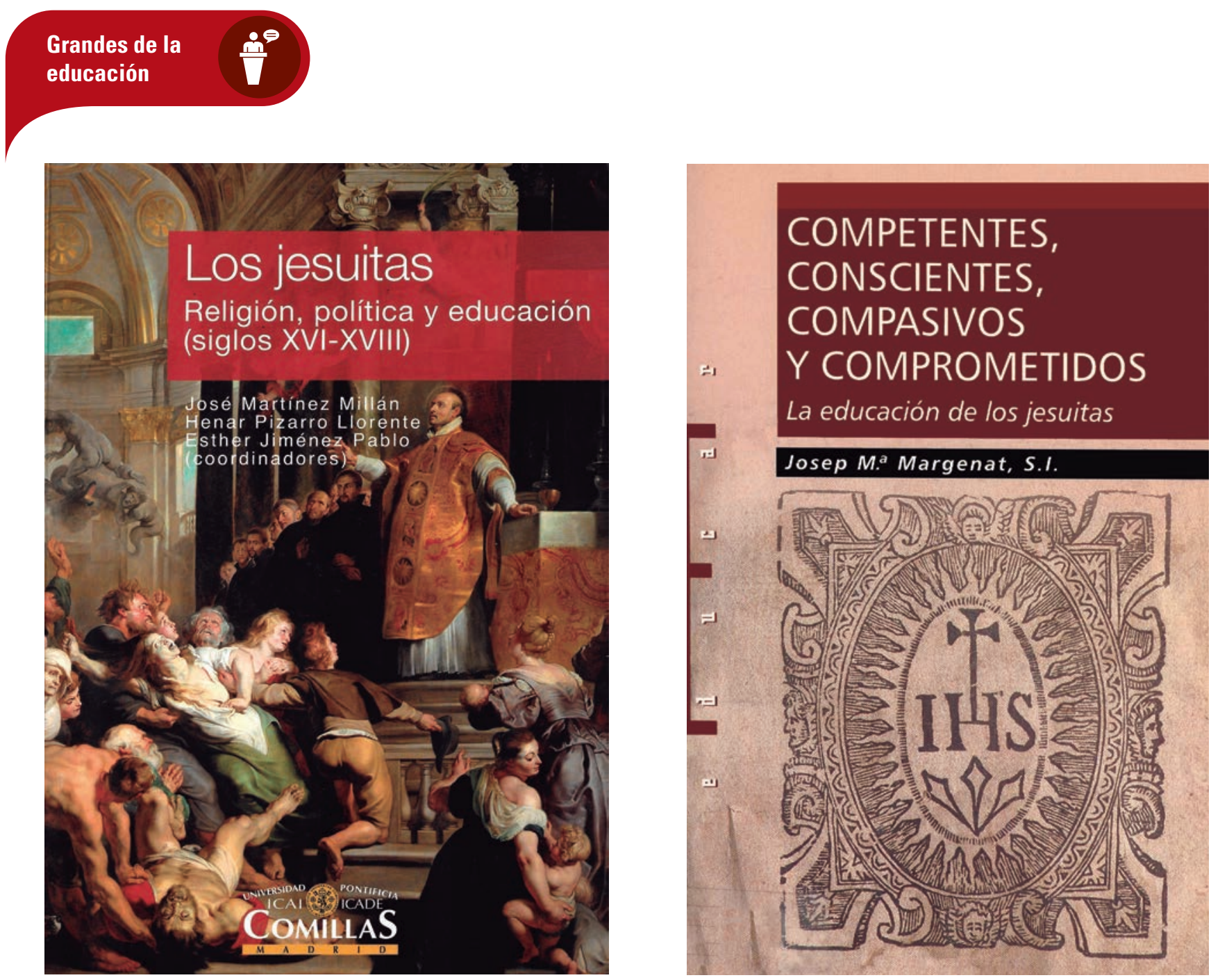

a "las cuatro C" y explicó y ahondó en su significado. Conscientes, puesto que además de conocerse a sí mismos por el cultivo de su capacidad de interiorización y de la vida espiritual, tienen un conocimiento consciente y experimental de los problemas que afectan a la sociedad. Competentes en el ámbito profesional, fruto de una excelente formación académica. Compasivos, lo que posibilita la apertura a la solidaridad. Comprometidos, porque, desde las premisas anteriores, procurarán desde la fe, honestamente y por medios pacíficos, impulsar las transformaciones sociales y políticas que procuren alcanzar la justicia [texto 1]. Ciertamente, el P. Kolvenbach procedió a actualizar los cuatro principios pedagógicos que el P. Ledesma formuló en el siglo XVI: utitilitas o enseñanza práctica, justitia o cívico-social, humanitas o humana-personal, y fides o religiosa.

La Congregación General 35 (2008), que eligió al P. Adolfo Nicolás como superior general, vino a insistir en la misión de servicio a la fe y la promoción de la justicia, pero introdujo nuevos conceptos en relación con el contexto global, las nuevas tecnologías, la ecología y la internacionalización. En consecuencia, P. Nicolás incidió en la renovación de la educación y, en sus intervenciones y escritos, reiteró que había que educar a los estudiantes para que puedan afrontar los retos del mundo actual, tan necesitado de fe, de justicia y de reconciliación, con una noción de universalidad,

profundidad e imaginación. Estas tres herramientas posibilitarán combatir la cultura contemporánea de la superficialidad del pensamiento [texto 2].

El P. Arturo Sosa fue elegido general de la Compañía de Jesús en la Congregación General 36 en 2016. En sus intervenciones y escritos ha insistido sobre los desafíos que se han de afrontar en el nuevo contexto donde se desarrolla la educación y a los que se debe de dar respuesta.

\section{Retos y oportunidades}

El libro publicado recientemente, cuya edición ha estado a cargo de José Alberto Mesa SJ, titulado La pedagogía ignaciana. Textos clásicos y contemporáneos sobre la educación de la Compañía de Jesús desde san Ignacio de Loyola hasta nuestros días, nos ofrece los jalones de una tradición centenaria que, en palabras de John O'Malley SJ, "no tomará nuestras decisiones, pero nos ofrece, creo, un punto de vista privilegiado desde donde las podemos tomar" (p. 438). Esta pertinente obra se ha convertido en un referente obligado y necesario para aquellos que estén interesados en dar respuesta a los desafíos que tiene la educación en la actualidad.

En el cambio de paradigma que vive el mundo actual, la educación jesuita, fiel a tu tradición, debe de evolucionar, por lo que, entre otras cuestiones, podemos extraer cinco aspectos que suponen retos, 
pero también oportunidades, que se han de afrontar y resolver. En primer lugar, el mundo globalizado y de creciente interdependencia condiciona que las decisiones que afectan al medioambiente, la economía y los derechos humanos tengan dimensiones planetarias, al igual que, en el reverso de la moneda, la eliminación de los factores que contribuyen a la generación de aislamiento, exclusión y marginación. Por ello, hemos de esforzarnos en educar para la cooperación, la solidaridad y la reconciliación.

Asimismo, y como consecuencia de esta globalización-mundialización, hemos de orientar los centros educativos para trabajar en red. Se trata de mantener el arraigo local, pero con una apertura a la cooperación internacional, que contribuya a generar una ciudadanía global que permita trabajar en solidaridad. En seguimiento de este impulso se han puesto en marcha diversas iniciativas entre las que conviene resaltar el Coloquio Internacional de Educación Secundaria Jesuita (ICJSE) en 2012, el Seminario Internacional de Pedagogía y Espiritualidad Ignacianas (SIPEI) en 2014 o el Congreso Internacional de Delegados de Educación de la Compañía de Jesús en 2017 [texto 3].

En tercer lugar, ha de sustentarse el mantenimiento de la identidad ignaciana de los centros educativos y la inspiración apostólica que la justifica. Esa cuestión resulta esencial en aquellos espacios geográficos donde la presencia de jesuitas se ha visto muy reducida. En este sentido, la colaboración más activa con los laicos iniciada décadas atrás

\section{«En respuesta a la globalización de la superficialidad, sugiero que necesitamos estudiar más profundamente el mundo cultural emergente de nuestros estudiantes y encontrar formas crealivas de promover la profundidad del pensamiento e imaginación» P. Adolfo Nicolás}

adquiere una nueva significación. Son diversos los documentos que abordan esta cuestión y que han cimentado la puesta en marcha de diversas iniciativas. Sirva como ejemplo el Máster en Pedagogía Ignaciana organizado y coordinado por EDUCSI y las Universidades de Deusto, Pontificia Comillas y Loyola Andalucía, que inicia su andadura en el curso 2019-2020.

El cuidado del medioambiente, encuadrado en la misión más amplia de reconciliación, supone un reto y una gran oportunidad. Algunos centros han incorporado la preocupación ecológica en su currículo, se ha adherido a un estilo de administración, se han diseñado medidas de ahorro energético, etc. La escuela se ha convertido en una herramienta didáctica para sensibilizar a las nuevas generaciones hacia la ecología e implicarles en el cambio de relación con el medioambiente.

\section{MÁSTER EN PEDAGOGÍA IGNACIANA}

\section{¿PARA QUIÉN?}

Dirigido a docentes de la Compañía de Jesús, responsables de educación y profesorado de instituciones que comparten la inspiración ignaciana.

METODOLOGIA SEMIPRESENCIAL.

Actividad permanente del alumnado y acompañamiento de profesor/a-tutor/a.

Sesiones presenciales en Madrid, Loyola y Manresa.

IDENTIDAD IGNACIANA

Un máster interdisciplinar de carácter práctico que responde a los retos actuales de la Educación, aunando la innovación y la sabiduría que ofrece la tradición educativa jesuita.
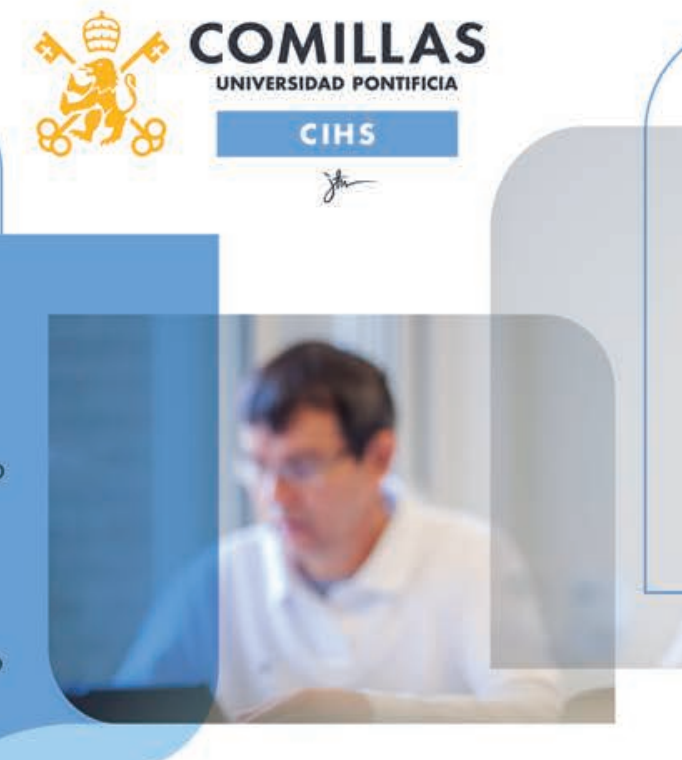

LA UNIVERSIDAD JESUITA DE MADRID

\section{COMILLAS}

CONTACTAR:

pedagogia.ignaciana@deusto.es 


\section{La acción educativa ha de orientarse a favorecer el crecimiento en la interacción de los grupos humanos y el respeto a sus diversas culturas como fórmula para capacitar una visión común de los intereses de toda la humanidad}

Por último, los cambios tecnológicos han traído nuevas formas de aprender y de enseñar a las nuevas generaciones. Esta cuestión no afecta únicamente a la incorporación de los nuevos recursos técnicos, como las pizarras digitales o los diversos dispositivos electrónicos, sino que interesa a la forma misma de entender la educación, la enseñanza y el aprendizaje, puesto que han contribuido a crear una nueva cultura que obedece a una nueva mentalidad. En consecuencia, se están desarrollando nuevos modelos educacionales no presenciales a través de internet, formatos semipresenciales, la ruptura de la tradicional relación de actividades para el alumno entre el hogar y el centro educativo, puesto que se ha invertido el aprendizaje que el alumno desarrolla en cada uno de estos espacios, se están implantado experiencias de "hiperaulas", donde confluyen varios profesores en un gran espacio en el que los alumnos se mueven libremente e incorporan el uso de la tecnología, etc.

En el contexto de universalización, donde debe encuadrarse la actual acción educativa, esta ha de orientarse a favorecer el crecimiento en la interacción de los grupos humanos y el respeto a sus diversas culturas como fórmula para capacitar una visión común de los intereses de toda la humanidad. La interculturalidad posibilitará la apertura a nuevos espacios. No en vano "el aporte espiritual de las religiones, entendidas como dimensiones de las culturas, propiciaría la superación de los fundamentalismos. Es lo que en 2008 intuyó la Congregación General 35 al invitarnos a ir a las fronteras de nuestras culturas y de la religión para encontrar, reconocer y entablar el diálogo con otros" [texto 4]. La universalidad así entendida puede convertirse en la senda que posibilite la justicia social, la fraternidad y la paz de los que depende el futuro Versión en inglés de la humanidad $•$

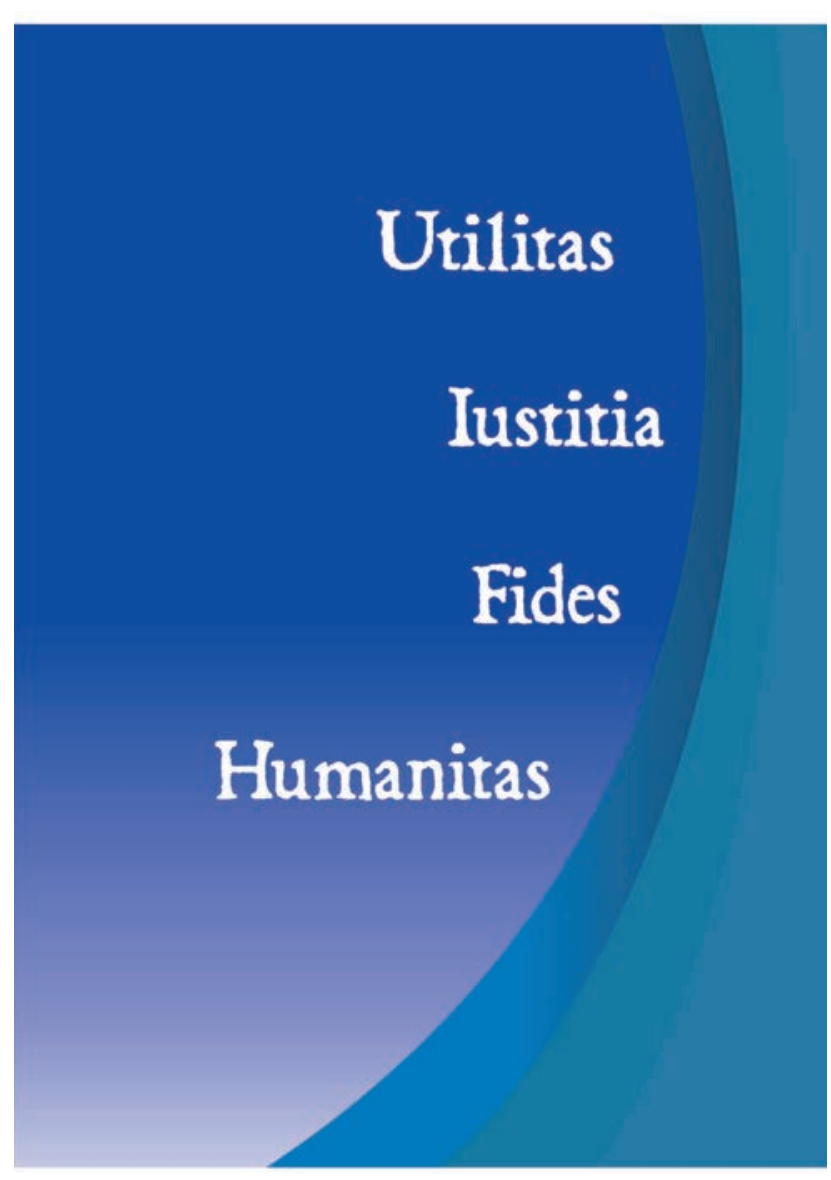

Todos los textos se han extraído del libro de José Alberto Mesa SJ (ed.) (2019). La Pedagogía ignaciana. Textos clásicos y contemporáneos sobre la educación de la Compañía de Jesús desde san Ignacio de Loyola hasta nuestros días. Mensajero-Sal Terrae-Universidad Pontificia Comillas (pp. 573, 582, 614, 628)

\section{DARA SABER MÂS}

CodinA, G. (1999). El "modo nuestro de proceder" en los estudios: la Ratio studiorum. Educatio S.J., 1,1-15.

GIL, E. (ed.) (2002). La pedagogía de los jesuitas ayer y hoy (2. ${ }^{a}$ ed.). Madrid: Universidad Pontificia Comillas.

Kolvenbach, P. H. (2007). Discursos universitarios. Selección e introducción por M. Agúndez. Madrid: UNIJES.

Mesa, J. A. (ed.) (2019). La Pedagogía ignaciana. Textos clásicos y contemporáneos sobre la educación de la Compañía de Jesús desde san Ignacio de Loyola hasta nuestros días. Mensajero-Sal Terrae-Universidad Pontificia Comillas.

Revuelta González, M. (1998). Los colegios de la Compañía de Jesús y su tradición educativa (1868-1906). Madrid: Universidad Pontificia Comillas.

Biblioteca. (2016). Pedagogía Jesuítica: de la Ratio Studiorum al proyecto educativo de Comillas. Universidad Pontificia Comillas. https://www.comillas.edu/es/noticias-biblioteca/9642-pedagogia-jesuitica-de-la-ratio-studiorum-al-proyecto-educativo-comillas 